\title{
NUCLEAR SPIN RELAXATION IN PERIODICALLY PERTURBED SYSTEMS IV. THE RELAXATION IN THE PRESENCE OF DOUBLE ROTATION AND PULSE SEQUENCE*
}

\author{
J.S. Blicharski, G. JASIŃski \\ Institute of Physics, Jagiellonian University \\ Reymonta 4, 30-059 Kraków, Poland \\ AND G. KLOSE \\ Fachbereich Physik der Universität Leipzig, Germany \\ (Received June 7, 1994)
}

\begin{abstract}
The effective relaxation times are calculated in the weak collision case for the system of identical nuclear spins perturbed by double rotation, periodic sequences of r.f. pulses and spin interactions.
\end{abstract}

PACS numbers: $76.60 .-\mathrm{k}$

\section{Introduction}

The aim of this paper is to extend the calculation of the effective relaxation times $T_{1 e}$ and $T_{2 \mathrm{e}}$ for a system of identical nuclear spins in the presence of the simultaneous perturbation by double rotation and periodic multi-pulse sequences.

In the previous papers [1-3] these two kinds of perturbations were considered separately. The influence of different pulse sequences on the effective spin-spin relaxation times was also presented in a series of papers [4-16].

\section{General theory}

Let us consider a system of identical nuclear spins $\boldsymbol{I}_{i}$ in a strong magnetic field $\boldsymbol{B}_{0}$ along $z$-axis, in the presence of double rotation around two axes $z_{1}, z_{2}$

*This work was supported by the Research Grant of the Committee for Scientific Research (Poland) and the DFG (Germany). 
at the frequencies $\omega_{1}, \omega_{2}$ and polar angles $\Theta_{1}=54.7^{\circ}$ and $\Theta_{2}=30.6^{\circ}$, respectively [5-7], and periodic train of very short pulses

$$
(\pi / 2)_{y}-\left[\tau_{0}-\left(\Theta_{1}^{\prime}\right)_{\Phi_{1}^{\prime}}-\tau_{1}-\left(\Theta_{2}^{\prime}\right)_{\Phi_{2}^{\prime}}-\tau_{2}-\ldots\left(\Theta_{N}^{\prime}\right)_{\Phi_{N}^{\prime}}-\tau_{N}\right]_{n}
$$

at resonance frequency $\omega_{0}=\gamma B_{0}$, where $\Theta_{n}^{\prime}$ and $\Phi_{k}^{\prime}$ are nutation and phase angles of the pulses with repetition period of the cycle $T_{\mathrm{c}}=\sum_{k=0}^{N} \tau_{k}$ and cycle frequency $\omega_{\mathrm{c}}=2 \pi / T_{\mathrm{c}}$.

The relaxation time $T_{\mathcal{Q}}$ for the expectation value $\langle\mathcal{Q}\rangle$ of an arbitrary spin operator $\mathcal{Q}$ in the weak collision case (WCC) can be calculated from the relation $[1-3]$

$$
\frac{1}{T_{\mathcal{Q}}}=\frac{\int_{-\infty}^{+\infty} \operatorname{Tr}\left\{[\mathcal{Q}, \tilde{\mathcal{H}}(t)][\mathcal{Q}, \tilde{\mathcal{H}}(t+\tau)]^{\dagger}\right\} \mathrm{d} \tau}{2 \operatorname{Tr}\left(\mathcal{Q} \mathcal{Q}^{\dagger}\right)}
$$

where $\tilde{\mathcal{H}}(t)$ is a time dependent Hamiltonian in an interaction frame (interaction representation).

In the presence of dipole-dipole interactions of like spins $1 / 2$ or axially symmetrical quadrupole interactions of spins $I=1$, the Hamiltonian in the laboratory frame has the following form:

$$
\begin{aligned}
& \mathcal{H}(t)=b \sum_{m=-2}^{+2} \mathcal{X}_{m}(t) \mathcal{T}_{2 m}^{\dagger}(I), \\
& \mathcal{X}_{m}(t)=b\left[\mathcal{Y}_{2 m}(\Theta(t) \Phi(t))-\left\langle\mathcal{Y}_{2 m}(\Theta(t) \Phi(t))\right\rangle\right],
\end{aligned}
$$

where $b$ is a coupling constant and $\mathcal{T}_{2 m}(\boldsymbol{I})$ and $\mathcal{Y}_{2 m}(\Theta, \Phi)$ are second rank spherical tensors and spherical functions, respectively.

All calculations have been done in the way described in the previous papers [1-3]. Using transformation properties for spherical tensors and spherical functions one gets the spin Hamiltonian in the interaction frame

$$
\tilde{\mathcal{H}}(t)=b \sum_{m m_{1} m_{2} m^{\prime}=-2}^{+2} \mathcal{D}_{m m_{1}}^{*}\left(\Omega_{1}\right) \mathcal{D}_{m_{1} M_{2}}^{*}\left(\Omega_{2}\right) \mathcal{D}_{m m^{\prime}}^{*}\left(\Omega^{\prime}(t)\right) X_{m_{2}}(t) \mathcal{T}_{2 m^{\prime}}^{\dagger}(\boldsymbol{I}),
$$

where

$$
\begin{aligned}
& \mathcal{D}_{m m^{\prime}}(\Omega(t))=\sum_{k=0}^{N-1} P_{k}(t) \mathcal{D}_{m m^{\prime}}\left(\Omega_{k}^{\prime}\right), \\
& \mathcal{D}_{m m^{\prime}}(\Omega) \equiv \mathcal{D}_{m m^{\prime}}^{(2)}(\alpha, \beta, \gamma)=\exp (-\mathrm{i} m \alpha) d_{m m^{\prime}}(\beta) \exp (-\mathrm{i} m \gamma) \\
& P_{k}(t)=\sum_{n=-\infty}^{+\infty} c_{k n} \exp \left(+\mathrm{i} n \omega_{\mathrm{c}} t\right),
\end{aligned}
$$

$P_{k}(t)$ are periodic square pulses with the width $\tau_{k} . \mathcal{D}_{m m^{\prime}}\left(\Omega_{k}\right)$ and $d_{m m^{\prime}}(\beta)$ are Wigner rotation matrices and Wigner functions, respectively, with $\Omega_{k}=(\alpha, \beta, \gamma) \equiv$ 
$\left(0, \Theta_{k}, \omega_{k} t\right)$ for $k=1,2$ and $\Omega_{k}^{\prime}=\left(\Phi-\frac{\pi}{2}, \beta_{k}, \frac{\pi}{2}-\Phi\right)$ in the case of r.f. pulses with a fixed phase $\Phi_{k}=\Phi$.

Using Eqs. (1-7) and commutation relations for $\mathcal{Q}=I_{z}$ and $I_{x}=\frac{1}{2}\left(I_{+}+I_{-}\right)$ one gets the following general expressions for the effective relaxation times in the case of non-oriented (powder) samples:

$$
\begin{aligned}
\frac{1}{T_{1 \mathrm{e}}}= & \frac{\Delta M_{2}}{6} \sum_{m m_{1} m_{2} m^{\prime} n} d_{m m^{\prime}}^{2}\left(\Theta_{1}\right) d_{m_{1} m_{2}}^{2}\left(\Theta_{2}\right)\left|C_{m m^{\prime}}^{n}\right|^{2}\left(m^{\prime}\right)^{2} \kappa_{m_{2}} \\
& \times \mathcal{J}_{m_{2}}\left(m_{1} \omega_{1}+m_{2} \omega_{2}-m \omega_{0}-n \omega_{\mathrm{c}}\right), \\
\frac{1}{T_{2 \mathrm{e}}}= & \frac{\Delta M_{2}}{12} \sum_{m m_{1} m_{2} m^{\prime} n}\left[\left|C_{m m^{\prime}}^{n}\right|^{2} \lambda_{m^{\prime}}+C_{m m^{\prime}}^{n} C_{m m^{\prime}+2}^{n *} \lambda_{m^{\prime}}^{+}+C_{m m^{\prime}}^{n} C_{m m^{\prime}-2}^{n} \lambda_{m^{\prime}}^{-}\right] \\
& \times \mathcal{J}_{m_{2}}\left(m_{1} \omega_{1}+m_{2} \omega_{2}-m \omega_{0}-n \omega_{\mathrm{c}}\right),
\end{aligned}
$$

where

$$
\begin{aligned}
& C_{m n^{\prime}}^{n}=\sum_{k} c_{k n} \mathcal{D}_{m m^{\prime}}\left(\Omega_{k}\right), \\
& \lambda_{m}=6-m^{2}, \\
& \lambda_{m}^{ \pm}=\frac{1}{2} \sqrt{(1 \mp m)(2 \mp m)(3 \pm m)(4 \pm m)},
\end{aligned}
$$

$\mathcal{J}(\omega)$ is the reduced spectral density of the correlation function in the presence of molecular motion with correlation time $\tau_{\mathrm{c}}$ :

$$
\mathcal{J}_{m}(\omega)=\int_{-\infty}^{+\infty} \frac{\left\langle X_{m}(t) X_{m}(t+\tau)\right\rangle}{\left\langle\left|X_{m}\right|^{2}\right\rangle} \mathrm{e}^{\mathrm{i} \omega \tau} \mathrm{d} \tau=\frac{2 \tau_{c}}{1+\omega^{2} \tau_{c}^{2}}
$$

and $\Delta M_{2}$ is the change of the second moment of the resonance line in the case of motional narrowing.

In a special case of a train of identical, equidistant r.f. pulses along $x$-axis with $\Theta_{k}^{\prime}=\Theta=2 \pi P / N, \Phi_{k}=0$ and $\tau_{k}=2 \tau$ for $k=1, \ldots,-N$ one gets

$$
\begin{aligned}
& c_{k n}=\frac{1}{n \pi} \sin \frac{n \pi}{N} \mathrm{e}^{-\mathrm{i} 2 \pi k n / N}, n \neq 0 ; c_{k 0}=\frac{1}{N}, \\
& \Omega_{k}=\left(\frac{\pi}{2},+k \Theta,-\frac{\pi}{2}\right) .
\end{aligned}
$$

The numerical simulations of the effective relaxation time $T_{2 \mathrm{e}}$ as a function of the correlation time $\tau_{\mathrm{c}}$ at different values of cycle frequency $\omega_{\mathrm{c}}$, and rotation frequencies $\omega_{1}, \omega_{2}$ and $\Theta=\frac{\pi}{2}$ are presented in Figs. 1, 2 and 3 respectively, showing minima of $T_{2 \mathrm{e}}$ at the region of $\omega_{\mathrm{c}} \tau_{\mathrm{c}}$ close to one. 


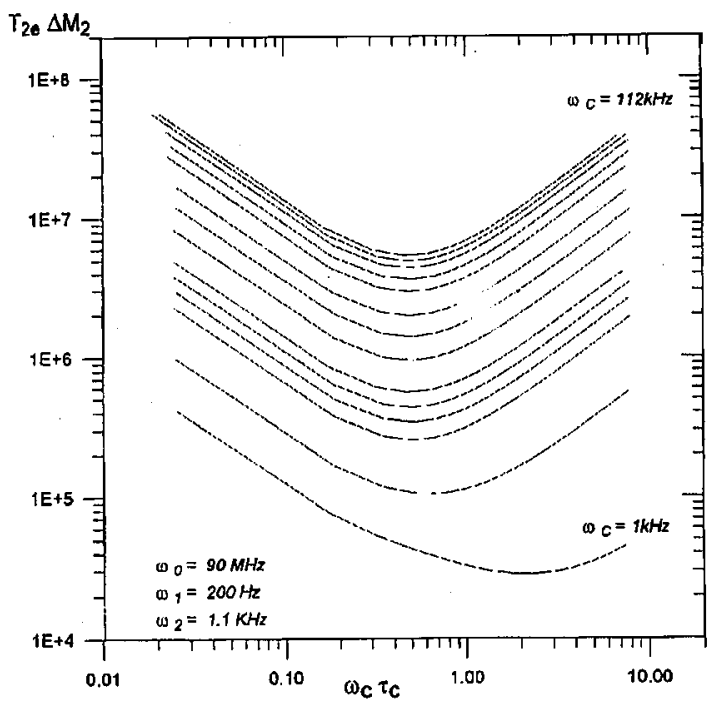

Fig. 1. $T_{2 c}$ as a function of the correlation time $\tau_{c}$ for $\omega_{0}=90 \mathrm{MHz}, \omega_{1}=200 \mathrm{~Hz}$, $\omega_{2}=1.1 \mathrm{kHz}$ and several values of $\omega_{\mathrm{c}}(112 \mathrm{kHz}, 102 \mathrm{kHz}, 91 \mathrm{kHz}, 75 \mathrm{kHz}, 61 \mathrm{kHz}$, $41 \mathrm{kHz}, 29 \mathrm{kHz}, 19 \mathrm{kHz}, 11 \mathrm{kHz}, 9 \mathrm{kHz}, 7.1 \mathrm{kHz}, 5.3 \mathrm{kHz}, 2.3 \mathrm{kHz}, 1 \mathrm{kHz}$ ).

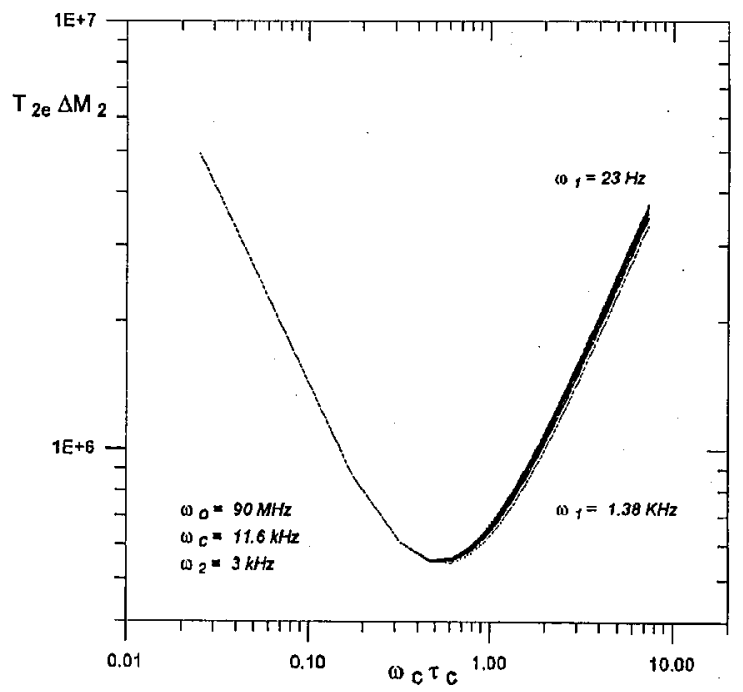

Fig. 2. $T_{2 \mathrm{e}}$ as a function of the correlation time $\tau_{c}$ for $\omega_{0}=90 \mathrm{MHz}, \omega_{\mathrm{c}}=11.6 \mathrm{kHz}$, $\omega_{2}=3 \mathrm{kHz}$ and several values of $\omega_{1}(23 \mathrm{~Hz}, 57 \mathrm{~Hz}, 95 \mathrm{~Hz}, 193 \mathrm{~Hz}, 313 \mathrm{~Hz}, 503 \mathrm{~Hz}$, $675 \mathrm{kHz}, 893 \mathrm{kHz}, 1 \mathrm{kHz}, 1.38 \mathrm{kHz})$. 
Nuclear Spin Relaxation in Periodically Perturbed Systems IV. .. 1005.

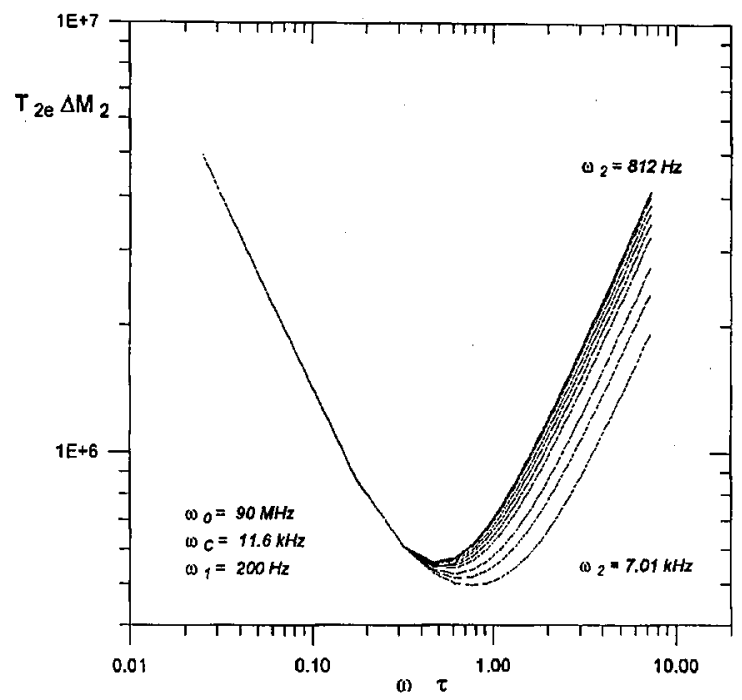

Fig. 3. $T_{2 \mathrm{e}}$ as a function of the correlation time $\tau_{\mathrm{c}}$ for $\omega_{0}=90 \mathrm{Mhz}, \omega_{\mathrm{c}}=11.6 \mathrm{kHz}$, $\omega_{1}=200 \mathrm{~Hz}$ and several values of $\omega_{2}(812 \mathrm{~Hz}, 1.1 \mathrm{kHz}, 1.7 \mathrm{kHz}, 2.5 \mathrm{kHz}, 3.1 \mathrm{kHz}$, $3.7 \mathrm{kHz}, 4.3 \mathrm{kHz}, 5.4 \mathrm{kHz}, 6.1 \mathrm{kHz}, 7.01 \mathrm{kHz})$.

\section{References}

[1] J.S. Blicharski, A. Wolak, Acta Phys. Pol. A 79, 591 (1991).

[2] J.S. Blicharski, A. Wolak, Acta Phys. Pol. A 82, 511 (1992).

[3] J.S. Blicharski, G. Jasiński, Acta Phys. Pol. A 82, 993 (1992).

[4] E.D. Ostroff, J.S. Waugh, Phys. Rev. Lett. 16, 1097 (1966).

[5] P. Mansfield, D. Ware, Phys. Lett. 22, 133 (1966).

[6] J.S. Waugh, C.H. Wang, Phys Rev. 162, 209 (1967).

[7] P. Mansfield, D. Ware, Phys. Rev. 168, 318 (1968).

[8] J.S. Waugh, L.M. Huber, U. Haeberlen, Phys. Rev. Lett. 20, 180 (1968).

[9] U. Haeberlen, J.S. Waugh, Phys. Rev. 185, 420 (1969).

[10] W. Grunde, H. Schmiedel, D. Freude, Ann. Phys. (Leipzig) 27, 409 (1971).

[11] P. Mansfield, Prog. Nucl. Magn. Reson. Spectrosc. 8, 43 (1971).

[12] A.N. Garroway, P. Mansfield, A.D. Stalker, Phys. Rev. B 11, 121 (1975).

[13] R. Muller, R. Wilsh, J. Mag. Resonance 21, 135 (1976).

[14] A. Jasiński, M. Stachurowa, Acta Phys. Pol. A 55, 433 (1979).

[15] W.K. Rhim, P. Burum, A.D. Eleman, J. Chem. Phys. 68, 692 (1978).

[16] A.J. Vega, R.W. Waughan, J. Chem. Phys. 68, 1958 (1978). 